\title{
ADVANCES IN ASTEROID AND SPACE DEBRIS SCIENCE AND TECHNOLOGY - Part 1
}

By Massimiliano Vasile

Asteroids and space debris represent a significant hazard for space and terrestrial assets; at the same time asteroids represent also an opportunity. In recent years it has become clear that the increasing population of space debris could lead to catastrophic consequences in the near term. The Kessler syndrome (where the density of objects in orbit is high enough that collisions could set off a cascade) is more realistic than when it was first proposed in 1978. Although statistically less likely to occur, an asteroid impact would have devastating consequences for our planet. Although an impact with a large $(\sim 10 \mathrm{~km})$ to medium $(\sim 300 \mathrm{~m})$ sized, or diameter, asteroid is unlikely, still it is not negligible as the recent case of the asteroid Apophis has demonstrated. Furthermore impacts with smaller size objects, between $10 \mathrm{~m}$ to $100 \mathrm{~m}$ diameter, are expected to occur more frequently and hence are, proportionally, equally dangerous for humans and assets on Earth and in space.

Asteroids and space debris share a number of commonalities: both are uncontrolled objects whose orbit is deeply affected by a number of perturbations, both have an irregular shape and an uncertain attitude motion, both are made of inhomogeneous materials that can respond unexpectedly to a deflection action, for both, accurate orbit determination is required, both need to be removed before they impact with something valuable for us. The desirable life cycle for both asteroids and space debris demonstrates that a number of underpinning technologies needs to be developed to mitigate the risk posed by both types of objects: observation, orbit determination, state estimation techniques are required to discover, identify, track and monitor both debris and asteroids. Impact prediction is required to inform decision makers and derive actions and requirements. Some form of manipulation, removal or deflection technologies/ strategies need to be developed and implemented and finally one can think of exploiting asteroids and needs to think of the consequences of disposing space debris (like the re-entry of large objects). The observation, manipulation and disposal of space debris represent one of the most challenging goals for modern space technology. It represents a key scientific and commercial venture for the future in order to protect the space environment for essential telecommunication, navigation and Earth observation services.

In order to address these issues, both ESA and the European Commission have created dedicated programs, like the Space Situational Awareness programme of ESA, or have invested significant funding to start new medium to long term research projects. This is part of a global effort that has seen other governments and organizations, in particular in the USA and Japan, initiating programs and initiatives aimed at resolving the asteroid and space debris issue. Among the recent projects supported by the European Commission, Stardust is a 4 year EU-wide programme, funded by the FP7 Marie Curie Initial Training Networks (ITN) scheme, which will train the next generation of engineers, scientists and decision makers to turn the threat represented by asteroids and space debris in an opportunity. Stardust will integrate multiple disciplines, from robotics, to applied mathematics, from computational intelligence to astrodynamics, to find practical and effective solutions to the asteroid and space debris issue

The first part of this special issue collects four papers on recent advances on the dynamics of artificial satellites in different orbit regimes around the Earth,followed by four papers on measurements, observations and orbit determination dedicated to space debris. Paper JASR 12158 then proposes an index to quantify the criticality of space objects and rank them according to their environmental impact. Three papers follow presenting strategies for the removal of spacecraft from libration points and high elliptical orbits, the active removal of space debris with harpoons and the detumbling of satellites exploiting eddy currents. The last four papers are dedicated to topics relevant to the mitigation of the threat from near-Earth objects and the manipulation of small bodies. 
All the articles submitted to this special issue went through the EES submission site (http://www.ees.elsevier.com/asr) and were regularly peer-reviewed by two to four reviewers.

The large number of submissions led us to publish this Special Issue in two separate parts. This first includes the papers that were accepted first. The second part is close to completion and will be published later this year in Advances in Space Research.

Finally, I would like to thank here all the authors and reviewers who made this ADVANCES IN ASTEROID AND SPACE DEBRIS SCIENCE AND TECHNOLOGY Special Issue a reality. As asteroids and space debris remain an open challenge in space science and engineering, I believe that this special issue will represent an important reference point for any future research on this compelling topic. 